\title{
LA AUTOMUTILACIÓN EN PERSONAS CON TRASTORNO LÍMITE DE LA PERSONALIDAD: ABORDAJE DE ENFERMERÍA EN UNA UNIDAD DE AGUDOS
}

\author{
MARIA ROMEU LABAYEN ${ }^{1,2}$, ANTONIO P. RAMAL MÁRQUEZ1 ${ }^{1}$ Y URTZI BLANCOU GAZTAÑAGA ${ }^{1}$ \\ ${ }^{1}$ Enfermera especialista en Salud Mental del Centre de Salut Mental d'Adults Horta Guinardó de Barcelona. \\ 2Profesora asociada del Departament d'Infermeria de Salut Pública, Salut Mental i Maternoinfantil \\ de la Universitat de Barcelona.
}

\section{INTRODUCCIÓN}

El trastorno límite de la personalidad (TLP) es un trastorno mental grave que afecta a un $2 \%$ de la población general y es el trastorno de la personalidad con mayor prevalencia. Las principales características son la inestabilidad en las relaciones personales y en la identidad, un alto nivel de impulsividad, autolesiones e ideación crónica de suicidio, ansiedad elevada, sufrimiento emocional y disminución de la motivación en las habilidades de resolución de problemas ${ }^{1}$. La desregulación emocional puede producir giros emocionales de tal intensidad que, en un breve espacio de tiempo, se alternan la euforia y la tristeza, pudiendo desembocar en una espiral de ansiedad y rabia en pocos minutos. Las crisis pueden producir consecuencias muy graves, incluso, letales ${ }^{2}$.

El trabajo para enfermería representa un reto en las unidades de hospitalización, debido a la falta de

Correspondencia: M. Romeu

Correo electrónico: maria.romeu1@gmail.com comprensión del trastorno y de la problemática que existe más allá de las conductas disruptivas, que, en muchas ocasiones, son la causa del ingreso. Esta es una dificultad importante, porque contribuye a mantener la percepción del paciente con TLP como desagradable, inconveniente y de difícil manejo ${ }^{3}$.

\section{PRESENTACIÓN DEL CASO}

Mujer de 28 años de edad, con diagnóstico psiquiátrico de TLP, que ingresa por cuarta vez en nuestra unidad de agudos tras sobreingesta medicamentosa (60 comprimidos de clotiapina y 20 comprimidos de levomepromazina de $25 \mathrm{mg}$ ) en el contexto de una ruptura sentimental. El tratamiento farmacológico al ingreso es: fluoxetina de $20 \mathrm{mg}$ (1-0-0); topiramato de 200 mg (1-0-1); olanzapina de 10 mg (0-0-1); y lormetazepam de 2 mg (0-0-0-1). En la valoración de enfermería al ingreso, se registró: buen aspecto físico, consciente y orientada en las tres esferas. En la entrevista, al principio, se muestra irritable y suspicaz, pero acaba rompiendo a llorar, manifestando desesperanza, anhedonia, disminución de la inges- 
ta, insomnio global, miedo a la soledad, sensación crónica de vacío e incertidumbre hacia su futuro personal.

Durante las primeras 24 horas, se mantiene aislada, cabizbaja, pensativa y clinofílica. Coincidiendo con el horario de visitas, solicita a un miembro del equipo una llamada telefónica para que le traigan sus enseres personales. Tras iniciar la conversación telefónica, la paciente se muestra vociferante, agitada y amenazante («me he tenido que ingresar por tu culpa [...]; si no vuelves conmigo, me mataré»). Lanza el teléfono contra la pared y se marcha corriendo a su habitación. Cuando el personal de enfermería llega a la habitación, la paciente presenta una contusión en la zona frontal y arañazos en ambos brazos y verbalizando: «no puedo más, me quiero morir», presentando tensión facial, temblores y sudoración intensa.

\section{DIAGNÓSTICOS}

Para el abordaje de la situación de crisis en la unidad de agudos, se realiza la valoración de enfermería (tabla 1), se formulan los siguientes diagnósticos, se establecen resultados y se describen intervenciones teniendo en cuenta la taxonomía NAN$\mathrm{DA}^{4}$, la clasificación de resultados $\mathrm{NOC}^{5}$ y la clasificación de intervenciones $\mathrm{NIC}^{6}$ (tabla 2):

1. Automutilación relacionada con (r/c) incapacidad para expresar verbalmente la tensión y manifestada por (m/p) contusión frontal y arañazos en los brazos.

Tabla 1. Valoración de las necesidades según el modelo de Virginia Henderson

\begin{tabular}{|c|c|}
\hline 1. Respirar & NPMD \\
\hline 2. Comer y beber & Disminución de la ingesta de alimentos \\
\hline 3. Eliminar & NPMD \\
\hline 4. Moverse & NPMD \\
\hline 5. Dormir y descansar & Insomnio global \\
\hline 6. Vestirse & NPMD \\
\hline 7. Mantener la temperatura & NPMD \\
\hline 8. Higiene & NPMD \\
\hline 9. Evitar peligros & Contusión frontal, arañazos en los brazos, amenaza con matarse \\
\hline 10. Comunicación & Llanto, irritabilidad, suspicacia, inquietud, tensión corporal, temblores \\
\hline 11. Actuar según los propios valores & Siente incertidumbre \\
\hline 12. Trabajar y realizarse & NPMD \\
\hline 13. Participar en el ocio & Anhedonia, sensación de vacío \\
\hline 14. Aprender & NPMD \\
\hline
\end{tabular}

NPMD: no presenta manifestaciones de dependencia. 
Tabla 2. Diagnósticos NANDA, resultados NOC e intervenciones NIC

\begin{tabular}{|c|c|c|}
\hline Diagnósticos NANDA & Resultados NOC & Intervenciones NIC \\
\hline Automutilación (00151) & $\begin{array}{l}\text { Autocontrol de la automutilación } \\
\text { (1406) } \\
\text { - No se autolesiona (140606) } \\
\text { (desde nunca demostrado = } 1 \text { hasta } \\
\text { siempre demostrado = 5) } \\
\text { Puntuación al ingreso: } 1 \\
\text { Puntuación al alta: } 4\end{array}$ & $\begin{array}{l}\text { Manejo de la conducta: autolesión } \\
\text { (4354) } \\
\text { - Retirar los objetos peligrosos del } \\
\text { entorno del paciente (435404) } \\
\text { - Disponer vigilancia continua del } \\
\text { paciente y de su entorno } \\
\text { (435406) } \\
\text { - Comunicar el riesgo a los demás } \\
\text { cuidadores (435407) } \\
\text { - Desarrollar expectativas y } \\
\text { consecuencias adecuadas de la } \\
\text { conducta, dado el nivel de la } \\
\text { función cognitiva y capacidad de } \\
\text { autocontrol del paciente } \\
\text { (435402) } \\
\text { - Animar al paciente a que hable } \\
\text { con los cuidadores cuando surja } \\
\text { el impulso de autolesionarse } \\
\text { (435412) }\end{array}$ \\
\hline & & $\begin{array}{l}\text { Cuidados de las heridas (3660) } \\
\text { - Monitorizar las características de } \\
\text { la herida, incluyendo drenaje, } \\
\text { color, tamaño y olor (366003) } \\
\text { - Administrar cuidados del sitio de } \\
\text { incisión, según sea necesario } \\
\text { (366008) }\end{array}$ \\
\hline Riesgo de suicidio (00150) & $\begin{array}{l}\text { Autocontrol del impulso suicida } \\
\text { (1408) } \\
\text { - Verbaliza control de impulsos } \\
\text { (140805) } \\
\text { (desde nunca demostrado= } 1 \text { hasta } \\
\text { siempre demostrado = 5) } \\
\text { Puntuación al ingreso = } 1 \\
\text { Puntuación al alta }=5\end{array}$ & $\begin{array}{l}\text { Manejo ambiental: prevención de } \\
\text { la violencia (6487) } \\
\text { - Eliminar las armas potenciales } \\
\text { del ambiente (648701) } \\
\text { - Controlar de forma sistemática el } \\
\text { ambiente para mantenerlo libre } \\
\text { de peligros (648702) } \\
\text { - Instruir a las visitas y los demás } \\
\text { cuidadores acerca de las } \\
\text { cuestiones relevantes sobre la } \\
\text { seguridad para el paciente } \\
\text { (648705) } \\
\text { - Instalar vigilancia continuada de } \\
\text { todas las zonas de acceso del } \\
\text { paciente para mantener su } \\
\text { seguridad e intervenir } \\
\text { terapéuticamente si es necesario } \\
\text { (648715) }\end{array}$ \\
\hline
\end{tabular}




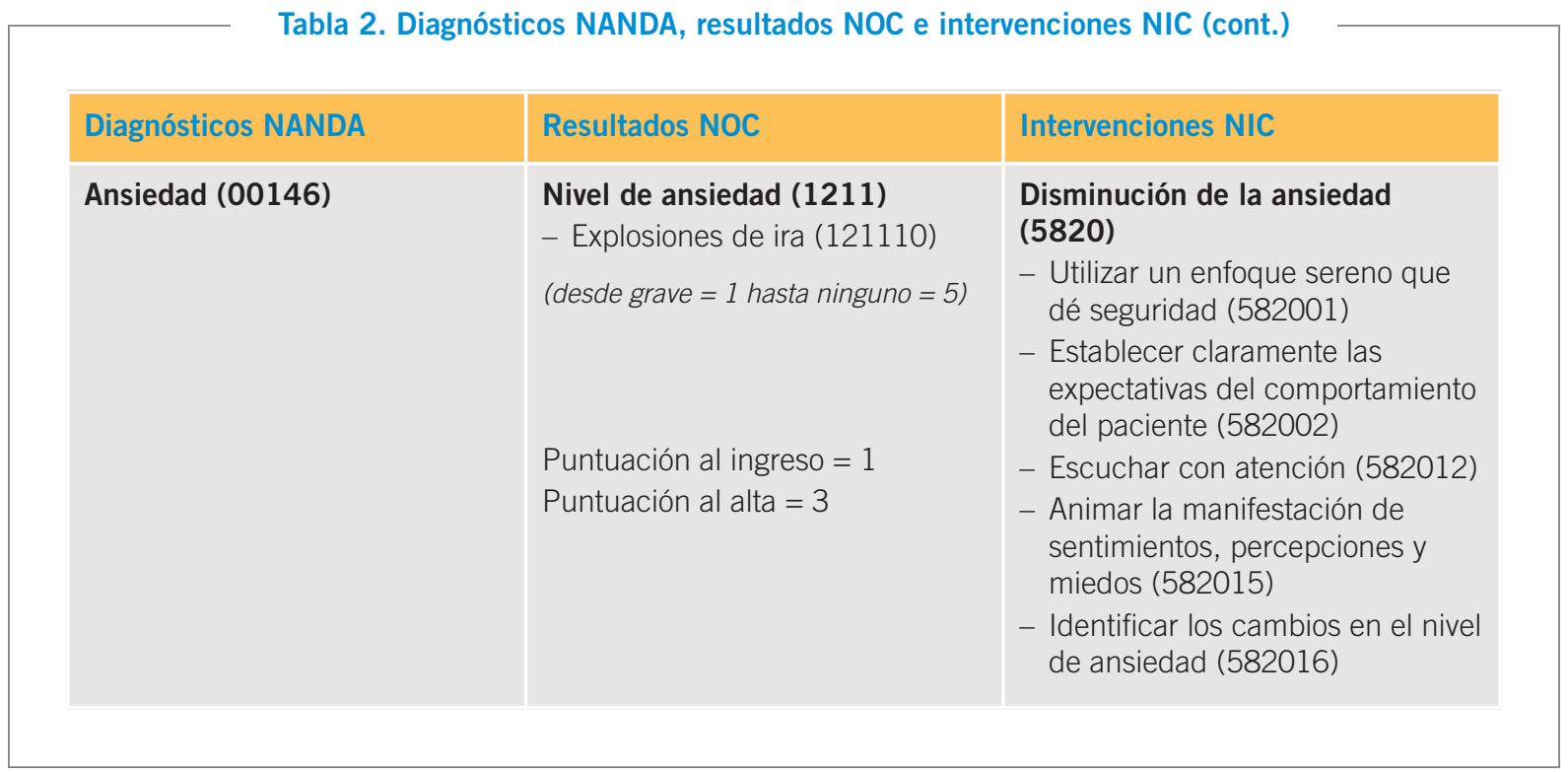

2. Riesgo de suicidio r/c amenaza con matarse.

3. Ansiedad $\mathrm{r} / \mathrm{c}$ amenaza para el autoconcepto y $\mathrm{m} / \mathrm{p}$ Ilanto, irritabilidad, inquietud, tensión, temblores, sudoración, disminución de la ingesta de alimentos e insomnio global.

\section{EVALUACIÓN}

El abordaje de la situación de crisis planteado se ha tenido activado hasta que la crisis ha cedido. La paciente ha estado acompañada en todo momento de la enfermera, que ha escuchado su malestar, ha facilitado la expresión de sus sentimientos y ha reflexionado con la paciente sobre la causa de la crisis y las dificultades de su gestión. La paciente ha podido reconocer el desencadenante de las crisis y ha verbalizado el deseo de aprender a solucionarlas evitando autolesionarse, siendo capaz de controlar los impulsos y su ansiedad e ira.

\section{BIBLIOGRAFÍA}

1. Pack S, Wakeham S, Beeby R, Fawkes L, Yeandle J, Gordon C. Management of borderline personality disor- der. Nurs Times. 2013;109(15):21-3. Disponible en: https://www. nursingtimes.net/download?ac $=1263490$

2. Borschmann R, Henderson C, Hogg J, Phillips R, Moran $\mathrm{P}$. Crisis interventions for people with borderline personality disorder. Cochrane Database Syst Rev. 2012;(6):CD009353. Disponible en:

http://onlinelibrary.wiley.com/doi/10.1002/14651858.C D009353. pub2/abstract;jsessionid=8BDD13C9A51F2 F3887B75DDDE30CDEAF.f04t04

3. Jiménez Barbero JA, Pérez García M, Rivera Rocamora C, Medina Garrido L, Munuera García V, Sánchez Muñoz M. El trastorno límite de personalidad: la filosofía dialéctica como base de la interacción enfermero paciente. Enfermería Glob. 2010;9(3). Disponible en: http://revistas.um.es/eglobal/article/view/111021/105421

4. NANDA International. Diagnósticos enfermeros. Definiciones y clasificación 2012-2014. Barcelona: Elsevier; 2013.

5. Moorhead S, Johnson M, Maas ML, Swanson E (eds.). Clasificación de Resultados de Enfermería (NOC). Medición de Resultados en Salud. 5. ${ }^{a}$ ed. Barcelona: Elsevier; 2014.

6. Bulechek GM, Butcher HK, Dochterman JM, Wagner C (eds.). Clasificación de Intervenciones de Enfermería (NIC). 6. ${ }^{a}$ ed. Barcelona: Elsevier; 2014.

7. Romeu Labayen M, Rigol Cuadra A, del Pino Gutiérrez A, Badenas Orts L, Miguel García C, Piñar Rodríguez $\mathrm{S}$. Proceso enfermero orientado a personas con trastorno límite de la personalidad. Barcelona: Dipòsit Digital de la Universitat de Barcelona Omado; 2015. Disponible en:

http://diposit.ub.edu/dspace/bitstream/2445/65878/ 1/Inf_SM_TLP.pdf 